\title{
USO DE DATOS EN LA CREATIVIDAD PUBLICITARIA: EL CASO DE ART, COPY \& CODE DE GOOGLE
}

\section{Use of data in advertising creativity: The case of Google's Art, Copy \& Code}

\section{David Selva-Ruiz y Lucía Caro-Castaño}
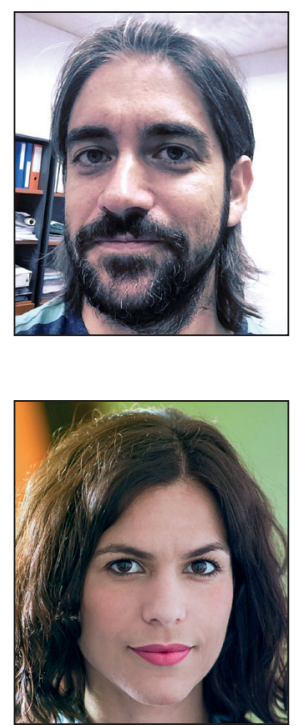

David Selva-Ruiz, doctor por la Universidad de Sevilla, es profesor del Departamento de Marketing y Comunicación y coordinador de Marketing e Imagen Institucional de la Universidad de Cádiz. Imparte clases sobre creatividad publicitaria y nuevas herramientas de comunicación comercial. Su investigación se centra en las nuevas tendencias y aplicaciones en comunicación y la cultura popular, contando con decenas de publicaciones en revistas científicas y libros académicos. Puede destacarse su libro El videoclip. Comunicación comercial en la industria musical (Alfar, 2014). http://orcid.org/0000-0002-9251-0045

david.selva@uca.es

Lucía Caro-Castaño es licenciada y doctora en publicidad y RRPP por la Universidad de Cádiz (UCA). Ha sido profesora asociada en la Universidad de Sevilla (US) y en la actualidad imparte docencia en el Departamento de Marketing y Comunicación de la UCA. Miembro del grupo de investigación Métodos, Análisis y Estrategias de la Comunicación Empresarial e Institucional (US). Investiga sobre nuevas prácticas y estrategias de comunicación online entre usuarios y marcas comerciales. Ha trabajado como responsable de comunicación en diversas empresas.

http://orcid.org/0000-0003-2720-1534

lucia.caro@uca.es

Universidad de Cádiz, Facultad de Ciencias Sociales y de la Comunicación Av. de la Universidad, s/n. 11405 Jerez de la Frontera (Cádiz), España

\section{Resumen}

La publicidad ha empleado tradicionalmente la investigación como una forma de conocer al consumidor, testar sus campañas, etc. Así, el uso de datos está relativamente asentado en la planificación estratégica publicitaria. En cambio, las posibilidades creativas de los datos están aún por explotar plenamente, máxime teniendo en cuenta que brindan oportunidades que la publicidad viene demandando, como una tendencia a la personalización y a la inmediatez. Este trabajo analiza el uso de datos en la creatividad publicitaria a través del caso del proyecto Art, copy \& code de Google y de cuatro de sus campañas. El análisis revela numerosos rasgos comunes, como la aportación de valor al usuario o el empleo conjunto de diversas tecnologías open web y varios dispositivos. Todo ello en el marco de procedimientos y estructuras asentados en el ámbito publicitario pero que se ven beneficiados de la aportación de los datos.

\section{Palabras clave}

Datos; Publicidad; Creatividad publicitaria; Comunicación digital; Marketing; Grandes datos; Big data; Datos abiertos; Open data; Publicidad online; Publicidad personalizada; Google.

\begin{abstract}
Advertising has traditionally used research as a way to know the consumer, test its campaigns, etc. Thus, data usage is already part of the strategic plan. However, the creative possibilities of data are yet to be fully exploited, especially those opportunities that provide personalization and immediacy. This paper analyzes data usage in advertising creativity through the case of Google's Art, copy \& code project and four of its campaigns. This analysis reveals many features that would add value for the user including joint use of various open web technologies and different devices, all within the framework of procedures and structures that are well-established in the advertising field.
\end{abstract}

\section{Keywords}

Data; Advertising; Advertising creativity; Digital communication; Marketing; Big data; Open data; Online advertising; Personalized advertising; Google. 
Selva-Ruiz, David; Caro-Castaño, Lucía (2016). "Uso de datos en creatividad publicitaria: el caso de Art, Copy \& Code de

Google". El profesional de la información, v. 25, n. 4, pp. 642-651.

http://dx.doi.org/10.3145/epi.2016.jul.14

\section{Introducción}

En el mercado publicitario Google ha sido hasta la fecha sinónimo de publicidad funcional: anuncios por palabras en su buscador y publicidad contextual en su red de espacios publicitarios. Aún hoy, pese a la diversificación de los formatos (apps de marca, publicidad nativa, etc.) la inversión en búsquedas pagadas mantiene el grueso de la inversión online en España -47,4\% en 2015 (IAB Spain, 2015)-. Sin embargo, muchas marcas necesitan no sólo estar presentes en el momento de la evaluación de compra, sino también tener una presencia social (Rayport, 2013) que les permita vincularse con su público objetivo. Art, Copy \& Code es un proyecto de Google destinado a fomentar el uso de la tecnología y en concreto de los datos en el ámbito publicitario y mercadotécnico, especialmente en las labores creativas. El objetivo último es posicionar a Google como una compañía clave en el sector de la publicidad, así como ubicar la gestión de los datos como un elemento tan importante como la redacción creativa y la dirección de arte en el día a día de la creatividad publicitaria en el entorno digital.

\section{Objetivos y metodología}

El presente trabajo tiene como objetivo examinar el uso de datos como recurso creativo en Art, Copy \& Code, mediante el análisis de los elementos comunes a las campañas de ese proyecto de Google. Ello permitirá conocer las características de esta tendencia publicitaria cada vez más presente en el panorama actual de la comunicación.

Hay que puntualizar que este no es un trabajo sobre Google en sentido estricto. Se elige el caso de Art, Copy \& Code como un proyecto paradigmático, en el que Google trata de abanderar lo que hoy es una tendencia creciente en publicidad, siendo un agente interesado, como Facebook y muchas otras empresas. El uso de datos es una opción más en el catálogo de recursos de los departamentos creativos de las agencias, y Google aspira a convertirse en socio prioritario de las agencias en este aprovechamiento de la tecnología y los datos.

Se lleva a cabo una revisión de los cambios producidos en la publicidad en relación con el uso de datos, y un análisis cualitativo de Art, Copy \& Code como caso paradigmático de esta tendencia publicitaria, y de cuatro de las cinco campañas que se exhiben en la web del proyecto.

http://www.artcopycode.com

Se ha excluido la quinta campaña (District Voices, de Lionsgate) dado que no se basa en el empleo de datos y escapa por tanto al objeto de estudio de este trabajo. Las cuatro campañas analizadas son:

- SmileDrive (Volkswagen)

- Kisses (Burberry)

- Phenomenal Shot (Nike)

- Madden Giferator (EA Sports).

\section{Uso de datos en creatividad publicitaria}

\subsection{El mensaje publicitario como contenido}

Transformar la publicidad en contenido elegido por el consumidor, eliminando su carácter intrusivo, ha sido un anhelo clásico de los profesionales de la publicidad. Para ello, a lo largo de la historia de esta disciplina, los equipos creativos han apelado al humor, al placer estético o a la emoción como recursos para atraer la atención del espectador (López, 2007). La llegada de la world wide web abrió un nuevo campo para la comunicación comercial y la exploración de nuevas formas de trasladar los mensajes de marca buscando superar el carácter intrusivo de los formatos tradicionales - dinámica push (spots, cuñas, gráficas, etc.)-, y potenciando la aparición de formatos que no interrumpiesen al usuario - bajo una dinámica pull (publicidad nativa, advergaming, apps, etc.)-.

Sin embargo, durante la primera época de internet, anunciantes y agencias se dedicaron a replicar el modelo intrusivo de los medios de comunicación de masas. El banner, en sus múltiples formatos (robapáginas, interstitials, layers, etc.), se convirtió inicialmente en la herramienta central de la publicidad online, aunque desde el principio los anunciantes expresaron su decepción por los bajos ratios de éxito del formato y la escasa información que ofrecían sobre el consumidor (Turow, 2011), un problema que persiste (Roca, 2014). El carácter intrusivo que perciben los usuarios en formatos como el banner (Van-Doorn; Hoekstra, 2013), así como el incremento de la publicidad nativa (Couldry; Turow, 2014), donde los mensajes promocionados adoptan las pautas formales de los contenidos informativos de la plataforma (los tweets patrocinados, por ejemplo), son factores que contextualizan que el principal problema en internet para los españoles siga siendo que encuentran "demasiada publicidad" - 59,4\%- (AIMC, 2016).

\section{Google aúna la mayor red de espacios publicitarios online con un abanico de servicios al usuario que le permite conocer tanto su grafo social, como su comportamiento de consumo}

Afortunadamente para anunciantes y publicitarios, en los comienzos del siglo XXI ha emergido una floreciente industria para la captación y análisis de grandes cantidades de datos (big data) sobre comportamiento de consumidor, así como un mayor conocimiento del modo en que los usuarios conciben este metamedio (Manovich, 2008) y el tipo de experiencias (Ashley; Tuten, 2015; Rayport, 2013; Martí; Muñoz, 2008) y relaciones (Sheehan; Morrison, 2009) que pueden ofrecer las marcas en estos entornos. 


\subsection{La personalización como nueva lógica del medio}

Consumir contenido en la Red implica al mismo tiempo producir datos (Deuze, 2006), dado que el actual diseño de la misma se caracteriza por la "dataficación" (datafication) de magnitudes nunca antes medidas sobre el comportamiento de los usuarios (Van-Dijck; Poell, 2013). Esta nueva fuente de datos permite la monitorización de las opiniones que los consumidores vierten sobre productos, marcas y campañas publicitarias.

Al convertir a todo usuario de la web 2.0 en un productor de contenido, la Red entró en un proceso de crecimiento exponencial de la información que, unido a la fragmentación de las audiencias y al crecimiento del consumo multipantalla (Nielsen, 2015), ha convertido la atención en un bien cada vez más escaso. En una encuesta realizada por Nielsen (2015) en 60 países, la consultora detectaba que:

- el $47 \%$ de los encuestados utiliza los medios sociales mientras ve televisión en directo;

- el 58\% usa internet para buscar contenidos relativos a lo que está viendo en televisión.

La personalización se perfila como la lógica mediática específica de internet al ofrecer a cada sujeto información relevante en relación con su propio consumo

La respuesta de compañías como Google, Amazon o Facebook a la necesidad de captar el interés de los usuarios ha sido la personalización de los contenidos, una adaptación que realizan de acuerdo con sus decisiones de consumo previas y/o con las elecciones de usuarios con intereses y comportamientos semejantes. Así, la personalización se perfila en la actualidad como la lógica mediática específica de internet (Couldry; Turow, 2014), al ofrecer a cada sujeto información relevante en relación con su propio consumo.

Los mensajes publicitarios personalizados son posibles gracias al refinamiento de los sistemas de intercambio y sincronización de cookies que permiten a las plataformas compartir estos archivos para rastrear los movimientos de un mismo usuario a través de la Web, aunque no se haya dado de alta (Nesamoney, 2015). Así, estas corporaciones pueden construir perfiles de consumidor cada vez más sofisticados que podrán alquilar a los anunciantes. A este empleo de los datos para mejorar las microsegmentaciones de perfiles debe añadirse la realización de pretests de optimización para conocer el éxito de las diferentes versiones de un mismo anuncio, lo que permite incrementar la eficacia de las campañas sobre la marcha (Nichols, 2013).

\subsection{Datos contextuales como fuente de relevancia para la publicidad}

Sheehan y Morrison (2009) plantearon la emergencia de una "cultura de la confluencia" en la web social, donde tendencias diversas tienden a fusionarse bajo la lógica del remix y el mashup. Esta cultura del remix ofrecería una nueva vía para resolver el desafío de la involucración que afronta- ba la publicidad online: reinventar el modelo de los mensajes masivos y ayudar al consumidor a contar historias. Al desarrollo de este tipo de narrativa propia del medio digital ha contribuido de modo central la mejora en la interoperabilidad entre plataformas, así como la implementación de nuevos estándares de diseño web abiertos - html5, CSS y Java Script, frente a Flash y ActionScript- que reducen la dependencia de la instalación de pluggins y permiten la combinación de datos de varios servicios en tiempo real (Percival, 2013). De este modo se ha ido configurando un ecosistema digital que ofrece nuevas libertades expresivas a la publicidad frente al encorsetamiento de los formatos en medios tradicionales, al tiempo que permite formas de incorporar al usuario en el propio mensaje. En esta línea, el publicitario español y socio fundador de la agencia interactiva española Double You, Daniel Solana (2012), ha señalado que el actual desarrollo de la Web ha permitido la aparición de una creatividad líquida, donde el soporte es sólo el punto de partida de la vinculación entre consumidor y marca.

Pese a que Solana anunciaba hace ya varios años la entrada en una nueva era de la creatividad online, la incorporación de los datos de modo creativo en los anuncios y proyectos de marca ha comenzado a desarrollarse en fechas muy recientes. La categoría Creative data, por ejemplo, no ha aparecido en los Cannes Lions hasta la edición de 2015. En este contexto de reinvención, la iniciativa Art, Copy \& Code de Google es probablemente la apuesta más potente del sector, en tanto que la compañía aúna el poder mediático, gracias a su capacidad para atraer la atención de los usuarios con:

a) la mayor red de espacios publicitarios (Google Display Network), que permite la puja en tiempo real (Papí-Gálvez, 2015) mediante la compra programática;

b) el acceso al grafo social de estos (Gmail, G+, Google Docs, etc.);

c) el rastreo de su comportamiento a través de los servicios que le presta (buscador, Google Chrome, YouTube, Google Play, etc.).

\section{La adaptación en tiempo real del anun- cio al contexto del consumidor median- te diferentes servicios de información es una estrategia eficaz para atraer su atención}

Otra forma de incrementar la relevancia potencial de los mensajes persuasivos es la incorporación de información contextual en tiempo real (Nesamoney, 2015; Rayport, 2013; Caro-Castaño; Selva-Ruiz, 2011). De este modo, por ejemplo, la adaptación del anuncio a la temperatura de la ciudad desde la que el usuario está conectado, la incorporación de la música que ha escuchado recientemente en una radio online o la aparición de la celebridad a la que sigue en Twitter, serían decisiones creativas que ayudan a atraer su atención. La incorporación de estos datos de contexto permite que el usuario se sienta interpelado, sin llegar a ser informaciones tan personales que deriven en una sensación 
de pérdida de privacidad (Tucker, 2014), como sucede con la incorporación de datos de transacciones previas (VanDoorn; Hoekstra, 2013).

Las ideas creativas y su desarrollo se apoyan en las posibilidades prestadas por la tecnología y en concreto por los datos

\section{El caso de Art, Copy \& Code de Google}

\subsection{El proyecto: introducción y contextualización}

Art, Copy \& Code es una iniciativa de Google a escala mundial en el campo publicitario, que se puso en marcha en 2013 y pervive en la actualidad. Como compañía especializada en productos y servicios de tecnología e internet, Google presta gran atención a la publicidad como elemento clave en su exitoso modelo de negocio. Por esta razón no debe sorprender que intente aliarse con este sector, y en concreto con el subsector de la creatividad publicitaria. El propio título de la iniciativa es ya una declaración de intenciones en toda regla. Desde los años 60 del siglo XX, el trabajo creativo tiene su núcleo en duplas creativas, sumando un art director o director de arte y un copy (copywriter) o redactor creativo. Esta estructura de trabajo, propuesta por Bill Bernbach (Castellblanque, 2006), figura clave en la historia de la publicidad, simboliza para Google una revolución creativa en el universo publicitario. Al insertar la palabra Code está ubicándose como un tercer elemento clave en la publicidad del futuro:

"Este proyecto fue inspirado por la revolución creativa de la década de 1960, cuando todo cambió simplemente asociando a directores de arte y redactores creativos. Había nacido la idea de un equipo creativo formado por 'arte y copy'. Hoy en día estamos en medio de una segunda revolución creativa, impulsada por la tecnología. El 'código' está siendo agregado al núcleo del proceso creativo. Nuestros proyectos de Art, Copy \& Code están orientados a proporcionar inspiración para todos aquellos que dan forma al futuro de la publicidad" (Google, 2016).

La novedad real de estas campañas se encuentra, no en el uso de datos para investigar al consumidor o evaluar resultados, aspectos que tocarían con otros ámbitos de la publicidad (planificación estratégica, planificación de medios, etc.), sino en el uso de los datos como activo para la creatividad. Son las propias ideas creativas y su implementación las que se apoyan en las posibilidades prestadas por la tecnología, y en concreto, por los datos.

El proyecto constituye un paso más allá en una estrategia que viene de atrás. Cabe resaltar el proyecto Re: Brief, de 2012. La propuesta, basada en la alianza con las marcas Coca-Cola, Volvo, Alka-
Seltzer y Avis, consistía en la actualización de una de sus campañas publicitarias icónicas. En colaboración con cada marca y con publicitarios que participaron en la creación de estas campañas, se adaptaban las campañas de publicidad convencional (basadas en anuncios convencionales en televisión, prensa, etc.) a las posibilidades brindadas por las nuevas tecnologías. Así por ejemplo, el mítico spot Hilltop (1971), de Coca-Cola, se reinventó con la colaboración del director de arte Harvey Gabor en una nueva campaña adaptada a las nuevas posibilidades prestadas por el medio digital, manteniendo el planteamiento estratégico y creativo de la campaña original pero añadiéndole personalización, participación del usuario, etc. $y$, en general, todos aquellos rasgos de la publicidad actual que eran inviables a principios de los años 70 del siglo XX. En concreto, el spot Hilltop, basado en la frase l'd like to buy the world a Coke y centrado en la celebración de la tolerancia y la unidad del género humano, se convierte con este proyecto en algo más. La nueva propuesta permite que los usuarios realmente "compren al mundo una Coca-Cola", de modo que pueden grabar su mensaje con su móvil o su ordenador y enviarlo a cualquier lugar del mundo junto con una Coca-Cola; posteriormente, un usuario desconocido recibirá dicho mensaje mediante una máquina de vending diseñada al efecto, desde la cual podrá responder al mensaje. En paralelo los usuarios pueden ver, descargar y compartir películas personalizadas con el viaje del mensaje y la Coca-Cola. Así, lo que era un spot de carácter unidireccional se convierte en una acción compleja donde el usuario es partícipe activo y vive una experiencia personalizada. El lema Advertising reimagined es bastante certero en cuanto al objetivo del proyecto, que coincide con el de Art, Copy \& Code.

Se puede comprobar cómo Google intenta mostrar su propuesta como una nueva etapa en la historia de la publicidad, que resulta de una evolución natural propiciada por las nuevas posibilidades prestadas por la tecnología. Art, Copy \& Code persigue, según Google:

"explorar nuevas formas en que la tecnología puede ayudar a construir marcas en un mundo digital. Desarrollamos campañas y experiencias que esperamos que la gente amará, recordará y compartirá" (Google, 2016).

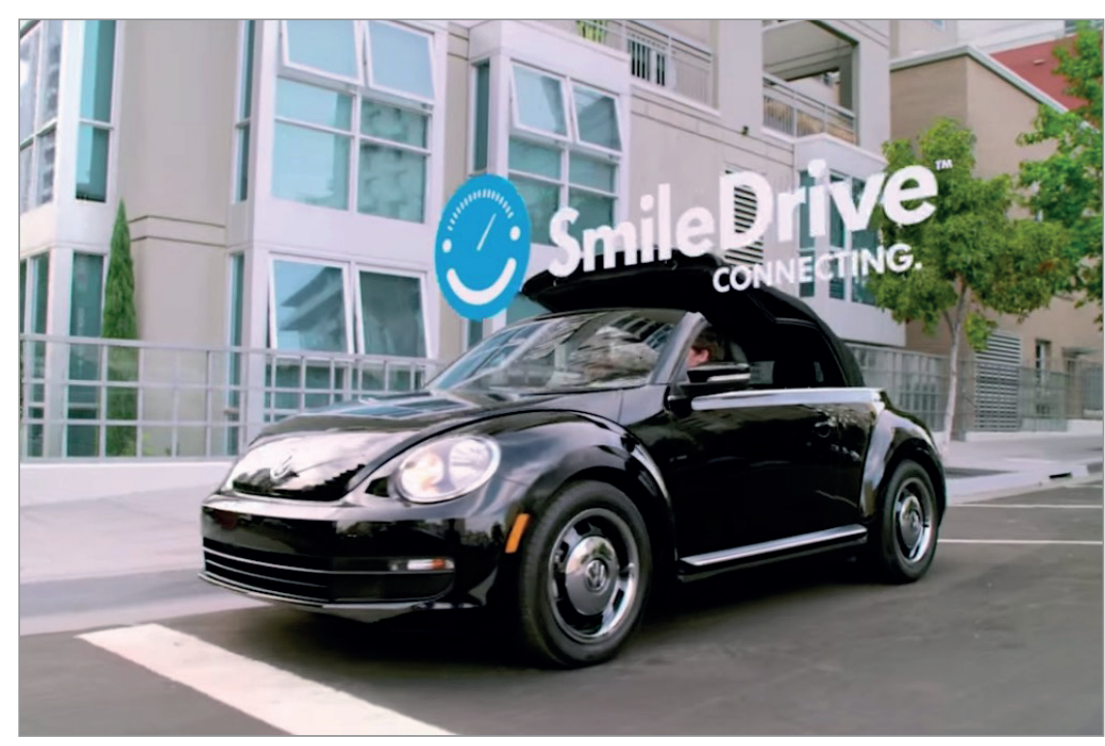

SmileDrive, para Volkswagen 

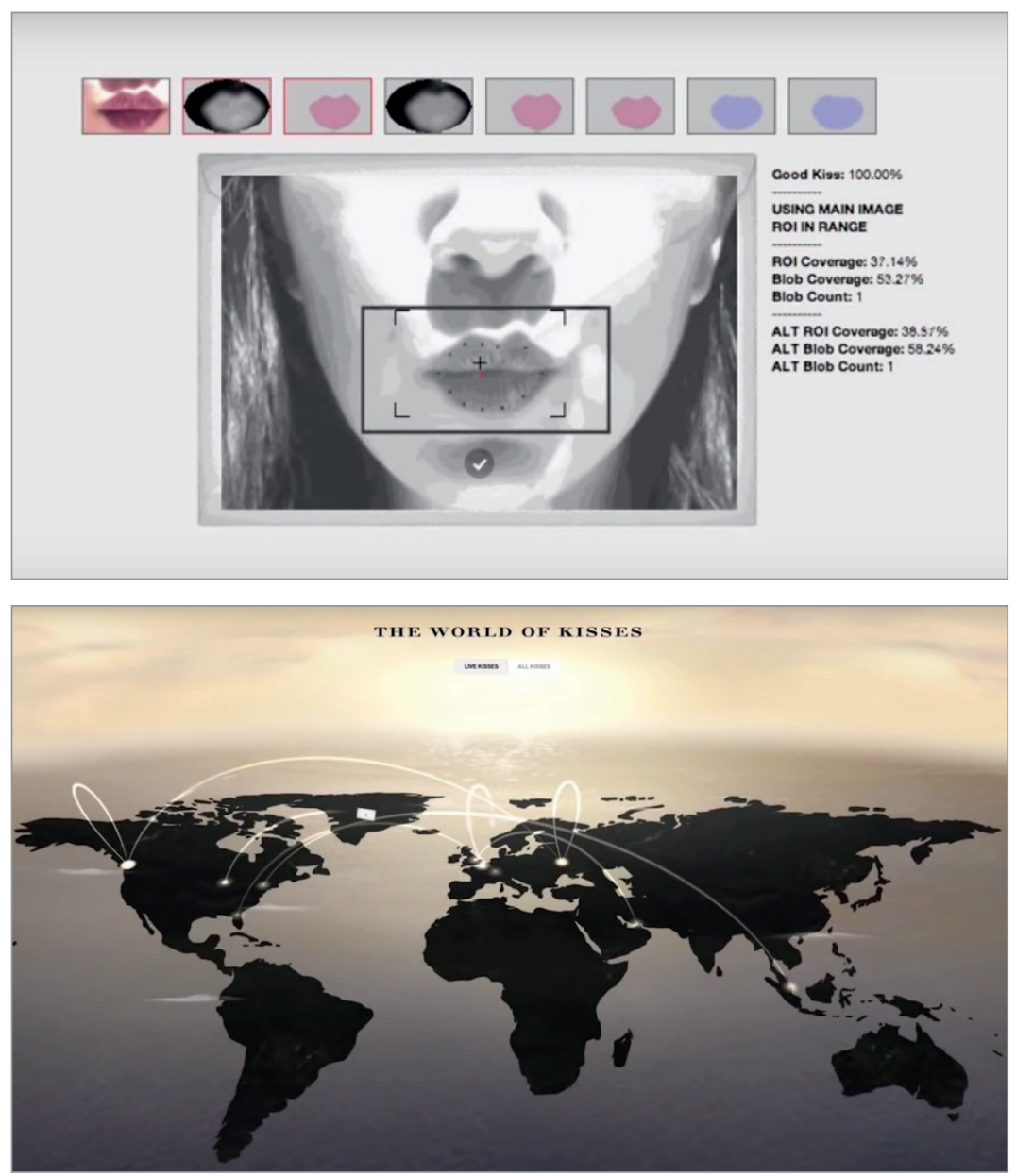

Kisses, para Burberry

\subsection{Descripción de las campañas}

\section{SmileDrive (Volkswagen)}

Consiste en una aplicación orientada a convertir la conducción en una experiencia social. Las personas pasan muchas horas conduciendo, la mayoría de ellas en soledad. La aplicación les permite compartir momentos y estar conectados con los demás de un modo seguro (la aplicación se cierra automáticamente cuando se enciende el motor). Toma datos de tráfico, meteorología, duración, localizaciones, etc. y permite crear un registro de fotos, mapas, actualizaciones de estado, etc., que incluso pueden ser retransmitidos en directo (SmileCast) mediante Google+. Al finalizar la conducción, proporciona al usuario todo ese material y una puntuación (SmileScore). Consiste prácticamente en convertir la conducción en una experiencia lúdica.

\section{Las cuatro campañas se basan en la cola- boración entre productos y tecnologías, todos ellos clasificables como open web technologies}

\section{Kisses (Burberry)}

Se trata de una campaña orientada a conectar emocionalmente con los millenials. Para ello se recurre a los besos, a la posibilidad de enviarlos virtualmente entre personas de todo el mundo y seguir su viaje. A través de una aplicación para móviles y tablets, se puede capturar el beso y enviarlo a quién se desee. Gracias a la conexión con los servicios de geolocalización de Google, se muestra visualmente el supuesto viaje de la carta con el beso entre el emisor y el receptor, incluyendo su vuelo por las calles de ambos.

\section{Phenomenal Shot (Nike)}

Intenta conectar con el público que está viendo retransmisiones deportivas desde la televisión. Partiendo de que en la actualidad el público, mientras visiona un partido, busca estadísticas en internet, comenta las jugadas, celebra las victorias, etc., se generan y difunden anuncios en tiempo real basados en jugadas que han sucedido sólo segundos atrás en el partido que se emite en televisión. Con el propio navegador (sin necesidad de descargar ninguna aplicación), el público puede girar 360 e en torno a la imagen hasta construir su propio anuncio Nike, que está siempre basado en un deportista vinculado a la marca.

\section{Madden Giferator (EA Sports)}

Tiene algunos puntos en común con la anterior. Partiendo de nuevo de un uso del móvil como segunda pantalla mientras se visiona un partido de la NFL (la liga estadounidense de fútbol americano) en la televisión, Madden Giferator crea una serie de gifs animados basados en lo que ha sucedido en el campo sólo segundos antes pero diseñados con imágenes propias del videojuego Madden NFL 15. Los gifs se muestran en tiempo real como anuncios, pero además el usuario puede editarlos mediante combinaciones de imágenes, frases y fondos.

\subsection{Aspectos comunes en las campañas}

De acuerdo con el análisis llevado a cabo, estas campañas se caracterizan por:

- importancia de los datos de cara a la ideación;

- personalización (automática) de la comunicación;

- vinculación con el experiential marketing;

- aportación de valor al usuario;

- mantenimiento de los conceptos básicos de la creatividad publicitaria;

- combinación entre comunicación push y pull;

- estructura de trabajo similar a la publicitaria (con el añadido de Google);

- cooperación entre distintos productos y tecnologías (open web technologies);

- uso de diversos dispositivos (con especial atención al móvil);

- vinculación con insights y conceptos cotidianos.

En todas las campañas analizadas, los datos tienen uno o varios roles de gran relevancia: conocimiento del consumidor, creatividad, segmentación en su difusión, evaluación 
de resultados, etc. El potencial de los datos es consabido en una industria como la publicitaria, que invierte una gran cantidad de recursos en investigación (González-Lobo; Prieto-del-Pino, 2009). Sin embargo, se insiste mucho por parte de Google en el potencial de los datos no sólo para definir la estrategia o evaluar resultados, sino también para la creatividad y, en particular, para el storytelling (técnica consistente en contar historias). Es un aspecto de tanta importancia para la compañía que el propio título de la misma parte de los dos principales roles creativos tradicionales, que se combinan con lo que defienden que debe ser un nuevo perfil fundamental en el trabajo creativo. Así, recomiendan que el equipo creativo trabaje en un mismo espacio que los expertos en tecnología:

“Esto permite a los creativos y a los tecnólogos trasladar el trabajo de uno a otro a la perfección o trabajar en conjunto. $Y$ a veces los problemas técnicos se pueden resolver con una solución creativa (por ejemplo, un poco de experiencia de usuario podría corregir un error técnico), o viceversa" (Google, 2016).

Google acentúa el potencial de los datos no sólo para definir la estrategia o evaluar resultados, sino también para la creatividad y, en particular, para el storytelling

El empleo de datos facilita además la personalización de la comunicación. Esta tendencia no es algo tan novedoso en publicidad y marketing (Peppers; Rogers, 1996) como otros aspectos del proyecto. A priori, que el usuario pueda seleccionar textos e imágenes y crear su propio gif con Madden Giferator no resulta tan innovador. Pero sí lo es la personalización llevada a cabo a través de los datos sin necesidad de una selección por parte del usuario. En SmileDrive, la propia aplicación va recopilando datos del viaje del usuario, de modo que el contenido personalizado que éste podrá ver, y que quizá incluso ha ido retransmitiendo en directo, no es seleccionado activamente por él. Incluso el vídeo promocional del propio proyecto Art, Copy \& Code realiza algo parecido. Se trata de una pieza que cambia según el usuario y su contexto. Al lanzar el vídeo, el programa recopila datos sobre la hora, el lugar, la meteorología o los eventos que han tenido repercusión recientemente, configurándose automáticamente un vídeo que supone una experiencia personalizada.

Desde un punto de vista mercadotécnico, las campañas de Art, Copy \& Code se ubican en la órbita del experiential marketing (Ashley; Tuten, 2015; Rayport, 2013; Martí; Muñoz, 2008; Cova; Salle, 2008). Éste se centra en la creación de experiencias para el consumidor en torno a la marca: enviar besos a quien el usuario desee en cualquier lugar del mundo, compartir gifs animados para burlarse de los seguidores del equipo rival, convertir la conducción en un juego, etc. Evidentemente, algo que tienen en común las cuatro marcas es que son de alto valor añadido y pertenecen a categorías de producto propicias para este tipo de estrategias.

\section{El experiential marketing se centra en la creación de experiencias para el consu- midor en torno a la marca}

Es importante destacar que las cuatro campañas analizadas se basan en aportar valor al usuario ofreciéndole una experiencia personalizada y un contenido entretenido o útil. No en vano el propio término "campaña" comparte protagonismo de forma creciente con el de "proyecto" en el ámbito profesional de la publicidad, dada la disolución de fronteras entre comunicación y producto y la tendencia a que la comunicación no consista ya en una combinación de anuncios convencionales si no en aportar contenidos y valor al usuario con acciones que superan el alcance la publicidad convencional (o, dicho de otro modo, de los anuncios en medios tradicionales) ${ }^{1}$. Lo mismo sucede con las propuestas de Art, Copy \& Code, que constituyen productos en sí mismos que el usuario puede consumir. Así lo defiende Google cuando recomienda pensar en el marketing como un producto:

“Complementa los anuncios mediante la creación de una plataforma como una aplicación o un producto que ofrezca utilidad y entretenimiento añadidos para los consumidores" (Google, 2016).

Una prueba de esta característica sería el hecho de que el $66 \%$ de los usuarios que descargan la aplicación de SmileDrive no son propietarios de un Volkswagen (Google, 2016).

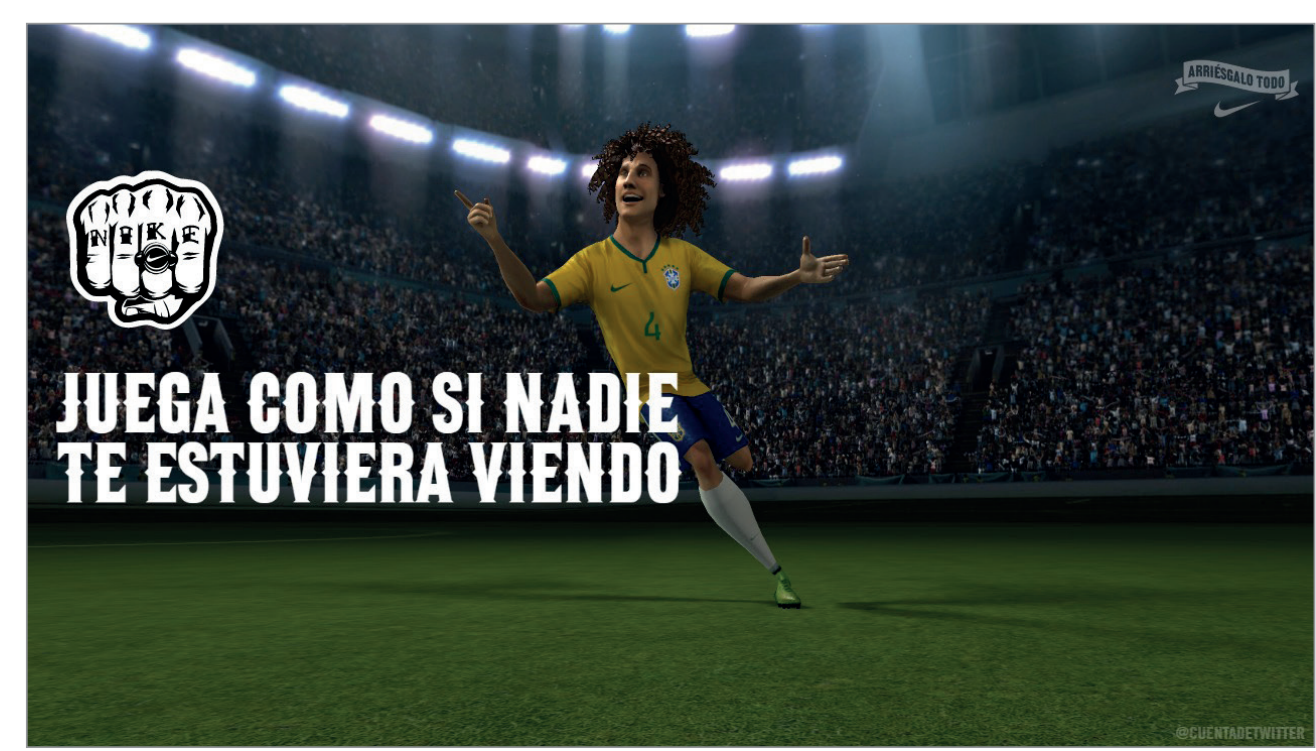

Imagen personalizada generada por Phenomenal Shot, para Nike 


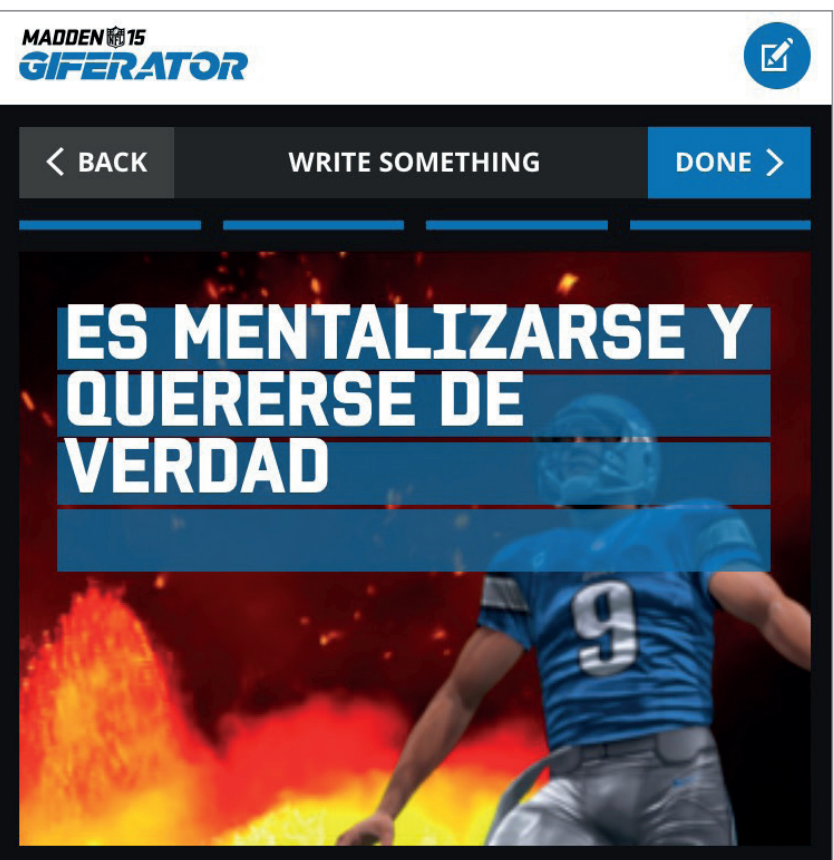

4 CHARACTERS REMAINING

I SCORE THEREFORE I DANCE

\section{GLAD I HAVE ME ON MY FANTASY TEAM}

MY TEAM BE LIKE.

TDS ALL UP IN YOUR ENDZONE

GAME OF THROWS

Captura de la pantalla del teléfono móvil durante la personalización en Madden Giferator, para EA Sports.

Es importante destacar que pese a las novedades, todo queda encuadrado en publicidad. Cuando se observa la explicación de Google respecto a las cuatro campañas analizadas, se puede comprobar que se siguen planteamientos que, con ciertos añadidos, vienen de años atrás: un brief, un insight, una big idea, etc. Evidentemente no deja de ponerse de relieve cómo los datos ayudan en cada uno de esos pasos. Por ejemplo, los big data pueden ser una fuente muy útil de cara a la detección de insights sobre el consumidor. Para Phenomenal Shot, los datos de Google mostraban los picos en búsquedas después de grandes momentos durante las competiciones deportivas, y eso resultó clave para partir del insight de que los usuarios emplean de forma intensiva su móvil como segunda pantalla justo después de esos grandes momentos. Así, Google propone "abrazar los datos":

Insight: Un aspecto oculto de la forma de pensar, sentir o actuar de los consumidores, útil para que la publicidad consiga conectar una marca con éstos y con sus experiencias cotidianas
"Los datos de búsquedas pueden ser una gran fuente de conocimiento acerca del tipo de contenido que los consumidores están buscando en grandes momentos culturales colectivos. El examen de estos datos puede ayudar, no sólo con lo que se crea, sino con cuándo se muestra al mundo" (Google, 2016).

Un aspecto de gran interés es que, pese a que las acciones tienen un marcado carácter de branded content y se basan en que el usuario elija acercarse a esos contenidos voluntariamente, las cuatro campañas de Art, Copy \& Code emplean también publicidad intrusiva (push). La cuestión es sencilla: se espera que la gente acuda voluntariamente al contenido, pero para eso el usuario debe conocer ese contenido, de modo que la publicidad push opera aquí como una muestra del mismo. Cierto es que no se emplean métodos extremadamente intrusivos, gracias al empleo de módulos Lightbox. Esto supone la inserción de un banner que, cuando el público permanece 2 segundos con el cursor sobre él, permite ver un vídeo o un contenido interactivo determinado de un modo más protagonista. Así sucede por ejemplo en SmileDrive, en cuyos anuncios Lightbox el público podía ver una muestra del funcionamiento de la aplicación en los casos de microcelebridades (blogueros, youtubers, etc.).

\section{Google muestra su propuesta como una nueva etapa en la historia de la publici- dad, que resulta de una evolución natu- ral propiciada por las posibilidades de la tecnología}

Por otra parte, se produce una segmentación extrema e inmediata gracias al uso de Google Display Network, que constituye una red de webs con espacio publicitario servido a través de Google Adwords. La existencia de esta red propia de Google permite que los anuncios aparezcan en las webs y aplicaciones de modo inmediato y en función de las búsquedas realizadas por el usuario a través de Google. Su uso es clave para Phenomenal Shot y para Madden Giferator, dado que las campañas se basan en la inmediatez con respecto a lo que sucede en los encuentros deportivos que se están emitiendo en televisión. Todo ello permite observar que:

- incluso en campañas tan novedosas como esas sigue empleándose publicidad intrusiva, dado que lo push y lo pull se retroalimentan;

- incluso lo push evoluciona para acercarse a las nuevas tendencias del usuario hasta convertirse prácticamente en un modelo mixto.

En la cadena de trabajo la gran novedad es la introducción de Google como sujeto activo en el proceso. No obstante, si la cadena tradicional para el desarrollo de un spot televisivo, estaba compuesta principalmente por un anunciante, una agencia y una productora audiovisual, cabe realizar una analogía entre esta última y el papel de Google en tanto que desarrollador. En cualquier caso, la estructura de trabajo no cambia tanto como cabría esperar, ni desde luego Google suplanta el rol de la agencia publicitaria, sino que se trata de una colaboración. Por ejemplo, en el caso de Kisses, tiene lugar una colaboración entre Burberry (anunciante), Grow 
(agencia digital) y Google (desarrollador). En otros casos, la estructura es más compleja, dadas las ramificaciones y complejidades técnicas de las campañas. Así, en Phenomenal Shot colaboran Nike (anunciante), Wieden+Kennedy (agencia publicitaria principal de la firma deportiva), Grow (agencia digital), Mindshare (agencia de medios) y Google (desarrollador).

Las cuatro campañas se basan en la colaboración entre productos y tecnologías, todos ellos clasificables como open web technologies. Exceptuando aquellos muy específicos de una campaña concreta, la mayoría de estas tecnologías son empleadas en varias campañas, y a su vez, cada campaña emplea un gran número de estas tecnologías (véase tabla 1). Por ejemplo en Phenomenal Shot, html5 y JavaScript operan de forma conjunta con datos obtenidos del giroscopio y la brújula del teléfono para determinar la posición del deportista en la imagen 3D generada, de modo que el usuario puede modificar su visión de aquel moviendo su teléfono móvil.

Además de tecnologías, se tienen en cuenta también los diferentes dispositivos. Por ejemplo Kisses puede emplearse en ordenadores, tablets y móviles, de modo que la experiencia interactiva sea semejante al margen del dispositivo que se use habitualmente. En cualquier caso existe un convencimiento por parte de Google de la importancia presente y futura del teléfono móvil. Esto se observa especialmente en Phenomenal Shot y en Madden Giferator, donde se parte de que los telespectadores del fútbol emplean constantemente el móvil como segunda pantalla para buscar información y estadísticas, comentar las jugadas, burlarse de los rivales, etc. Aprovechan la actividad del usuario y se adaptan a ella, algo que explica Google:

"Al crear herramientas y contenido para la segunda pantalla, recuerda que eres la cheerleader -chica animadora-, no el quarterback -líder ofensivo en el fútbol americano-. En lugar de campañas totalmente nuevas, distrayendo la acción, haz 'compañeros': maneras de alimentar la pasión sin interponerte en el camino" (Google, 2016).

Esta visión no implica una merma de las posibilidades del móvil: al contrario, Google defiende su importancia hasta expresar que

"para las pantallas pequeñas, pienses en grande" (Google, 2016).

Es decir, no conviene concebir el móvil como una versión en miniatura del escritorio del ordenador, sino que es factible exprimir posibilidades del móvil sin siquiera requerir la descarga de una app específica. Van más allá, planteando incluso la conveniencia de diseñar pensando en las pantallas pequeñas para luego realizar la adaptación a pantallas grandes, y no al contrario.

En todas las innovaciones suelen detectarse ideas sencillas y cotidianas, pero impulsadas gracias a las posibilidades tecnológicas. Es claro el ejemplo de Kisses, que como afirma Christopher Bailey, CEO de Burberry, parte de una idea muy simple:

"mandas uno de tus besos personales a cualquiera, en cualquier lugar del mundo, y sigues el viaje de tu beso" (Google, 2016).

Luego la tecnología lo hará posible, pero la base es algo tan sencillo como enviar besos. Según Drew Ungvarsky, director creativo de la agencia Grow, el éxito de la campaña viene precisamente de la combinación de ideas nuevas y antiguas, tomando un tema universal e introduciendo la tecnología en él (Google, 2016). El consejo de Google en relación con la campaña SmileDrive es identificar lo que hace especial a la marca, y sólo después añadir interactividad para crear una experiencia digital en torno a ello. 


\section{Conclusiones}

Como se ha podido comprobar en el caso de Art, Copy \& Code de Google, las posibilidades de uso de datos para la creatividad publicitaria son enormes, hasta el punto de que al estar aún en un estadio incipiente, resultan difíciles de imaginar en toda su dimensión.

Aun así, hay ciertos aspectos que merece la pena hacer notar. La personalización es una tendencia ya asentada en comunicación; sin embargo, la posibilidad de captar datos del usuario y la realidad que le rodea en tiempo real permite una automatización de esta personalización que podría mejorar sensiblemente la eficacia de las campañas, además de permitir el desarrollo de propuestas creativas que hace pocos años habrían resultado inviables. Ello se lleva cabo mediante la cooperación necesaria entre diversos productos y tecnologías (que en el caso del proyecto analizado son siempre open web technologies) y múltiples dispositivos. En otras palabras, existe un background técnico de gran complejidad, pero con la pretensión de que el acceso del usuario a la experiencia sea fácil, cómodo y lúdico.

\section{La posibilidad de captar datos del usuario} y de la realidad que le rodea en tiempo real permite una automatización de la personalización que podría mejorar sensiblemente la eficacia de las campañas

Hay que destacar que nos movemos en el entorno del experiential marketing y en la aportación de valor al usuario. Esto, que puede ser considerado una virtud, nos revela también su posible restricción a marcas de alto valor añadido. Junto con los debates que se suelen generar en torno a la privacidad en el uso de datos, este aspecto constituye una de las principales limitaciones de este fenómeno.

En cualquier caso se comprueba que los datos vienen a sumarse a planteamientos consolidados en publicidad, y no a sustituirlos. La interrelación entre comunicación push y pull, así como la evolución de la primera, el trabajo en una estructura similar a la publicitaria, a la que Google y los técnicos vienen a sumarse y no a reemplazar, o la vinculación con conceptos arraigados en la práctica profesional de la publicidad, demuestran que no existe una ruptura sino una evolución.

Las nuevas posibilidades tecnológicas y en concreto el uso de los datos permiten nuevas opciones para conectar con un usuario cada vez más huidizo y crítico con la publicidad tradicional y, por tanto, constituyen un elemento de gran relevancia para la comunicación comercial y con un potencial aún por desarrollar en su plenitud.

\section{Nota}

1. Pese a las diferencias conceptuales de ambas expresiones, campaña y proyecto se utilizan de forma casi indistinta en la práctica profesional de la publicidad. Ambas aludirían a iniciativas que son ideadas, planificadas y ejecutadas con un determinado objetivo de comunicación o publicitario, con el matiz de que la primera es la denominación tradicio- nal, mientras que la segunda se considera más abierta a acciones que desbordan la publicidad convencional, es decir, los anuncios.

\section{Bibliografía}

AIMC (2016). 18ㅇ Informe navegantes en la Red. Encuesta AIMC a usuarios de internet.

http://www.aimc.es/-Navegantes-en-la-Red-.htm/

Ashley, Christy; Tuten, Tracy (2015). “Creative strategies in social media marketing: An exploratory study of branded social content and consumer engagement". Psychology \& marketing, v. 32, n. 1, pp. 15-27.

http://dx.doi.org/10.1002/mar.20761

Caro-Castaño, Lucía; Selva-Ruiz, David (2011). “Estrategias de convergencia y desintermediación en la difusión del videoclip mediante autocomunicación de masas". Trípodos, n. extra, pp. 155-163.

Castellblanque, Mariano (2006). Perfiles profesionales de publicidad y ámbitos afines. Barcelona: Editorial UOC. ISBN: 8497885074

Couldry, Nick; Turow, Joseph (2014). “Advertising, big data and the clearance of the public realm: Marketers' new approaches to the content subsidy". International journal of communication, n. 8, pp. 1710-1726.

http://ijoc.org/index.php/ijoc/article/view/2166/1161

Cova, Bernard; Salle, Robert (2008). "Marketing solutions in accordance with the SD logic: Co-creating value with customer network actors". Industrial marketing management, v. 37, n. 3 , pp. $270-277$.

http://dx.doi.org/10.1016/j.indmarman.2007.07.005

Deuze, Mark (2006). "Participation, remediation, bricolage: Considering principal components of a digital culture". The information society, v. 22, n. 2, pp. 63-75.

http://jtc468.colostate.edu/docs/bricolage.pdf http://dx.doi.org/10.1080/01972240600567170

González-Lobo, María-Ángeles; Prieto-del-Pino, María-Dolores (2009). Manual de publicidad. Madrid: ESIC. ISBN: 978 8473566285

Google (2016). Art, Copy \& Code. A series of projects to reimagine the future of advertising.

http://www.artcopycode.com/campaign/art-copy-code

IABSpain (2015). Inversión publicitaria en medios digitales. Resultados 2015.

http://www.iabspain.net/wp-content/uploads/ downloads/2016/03/Estudio_Inversión_Publicitaria_Medios_ Digitales_2015_IAB_Spain_vReducida.pdf

López, Belén (2007). Publicidad emocional: estrategias creativas. Madrid: ESIC. ISBN: 9788473564885

Manovich, Lev (2008). Software takes command. New York: Georgetown University.

http://faculty.georgetown.edu/irvinem/theory/ManovichSoftware-Takes-Command-ebook-2008-excerpt.pdf

Martí, José; Muñoz, Pablo (2008). Engagement marketing. Una nueva publicidad para un marketing de compromiso. Madrid: Pearson Educación. ISBN: 9788483224496 
Nesamoney, Diaz (2015). Personalized digital advertising: How data and technology are transforming how we market. Old Tappan: Pearson Education. ISBN: 9780134030104

Nichols, Wes (2013). "Advertising analytics 2.0". Harvard business review, v. 91, n. 3, pp. 60-68.

https://hbr.org/2013/03/advertising-analytics-20

Nielsen (2015). Screen wars. The battle for eye space in a TV-everywhere world.

http://www.nielsen.com/content/dam/corporate/us/en/ reports-downloads/2015-reports/nielsen-global-digitallandscape-report-march-2015.pdf

Papí-Gálvez, Natalia (2015). “Nuevos medios y empresas innovadoras. El caso de las agencias de medios". El profesional de la información, v. 24, n. 3, pp. 301-309.

http://dx.doi.org/10.3145/epi.2015.may.10

Peppers, Don; Rogers, Martha (1996). The one to one future. Building relationships one customer at a time. New York: Currency. ISBN: 0385485662

Percival, John (2013). HTML5 advertising. New York: Springer Sciences+Bussines Media. ISBN: 9781430246022

Rayport, Jeffrey F. (2013). “Advertising's new medium: human experience". Harvard business review, v. 91, n. 3, pp. 76-82. https://hbr.org/2013/03/advertisings-new-medium-humanexperience

Roca, Meritxell (2014). "La transformación de la industria publicitaria en la era digital. Percepciones de los profesionales en Estados Unidos: retos y oportunidades". Telos: cua- dernos de comunicación e innovación, n. 99, pp. 64-74. https://telos.fundaciontelefonica.com/seccion=1268\&idioma=es_ ES\&id $=2014102812250001$ \&activo $=6$.do

Sheehan, Kim-Bartel; Morrison, Deborah K. (2009). "The creativity challenge: media confluence and its effects on the evolving advertising industry". Journal of interactive advertising, v. 9, n. 2, pp. 40-43.

http://dx.doi.org/10.1080/15252019.2009.10722154

Solana, Daniel (2012). Postpublicidad. Reflexiones sobre una nueva cultura publicitaria en la era digital. Barcelona: Doubleyou. ISBN: 8461405846

Tucker, Catherine E. (2014). "Social networks, personalized advertising, and privacy controls". Journal of marketing research, v. 51, n. 5, pp. 546-562.

http://papers.ssrn.com/sol3/papers.cfm?abstract_ $i d=1694319$

http://dx.doi.org/10.1509/jmr.10.0355

Turow, Joseph (2011). The daily you. How the new advertising industry is defining your identity and your worth. New Haven/London: Yale University Press. ISBN: 9780300165012

Van-Dijck, José; Poell, Thomas (2013). “Understanding social media logic". Media and communication, v. 1, n. 1, pp. 2-14. http://ssrn.com/abstract=2309065

Van-Doorn, Jenny; Hoekstra, Janny C. (2013). "Customization of online advertising: The role of intrusiveness". Marketing letters, v. 24, n. 4, pp. 339-351.

http://dx.doi.org/10.1007/s11002-012-9222-1

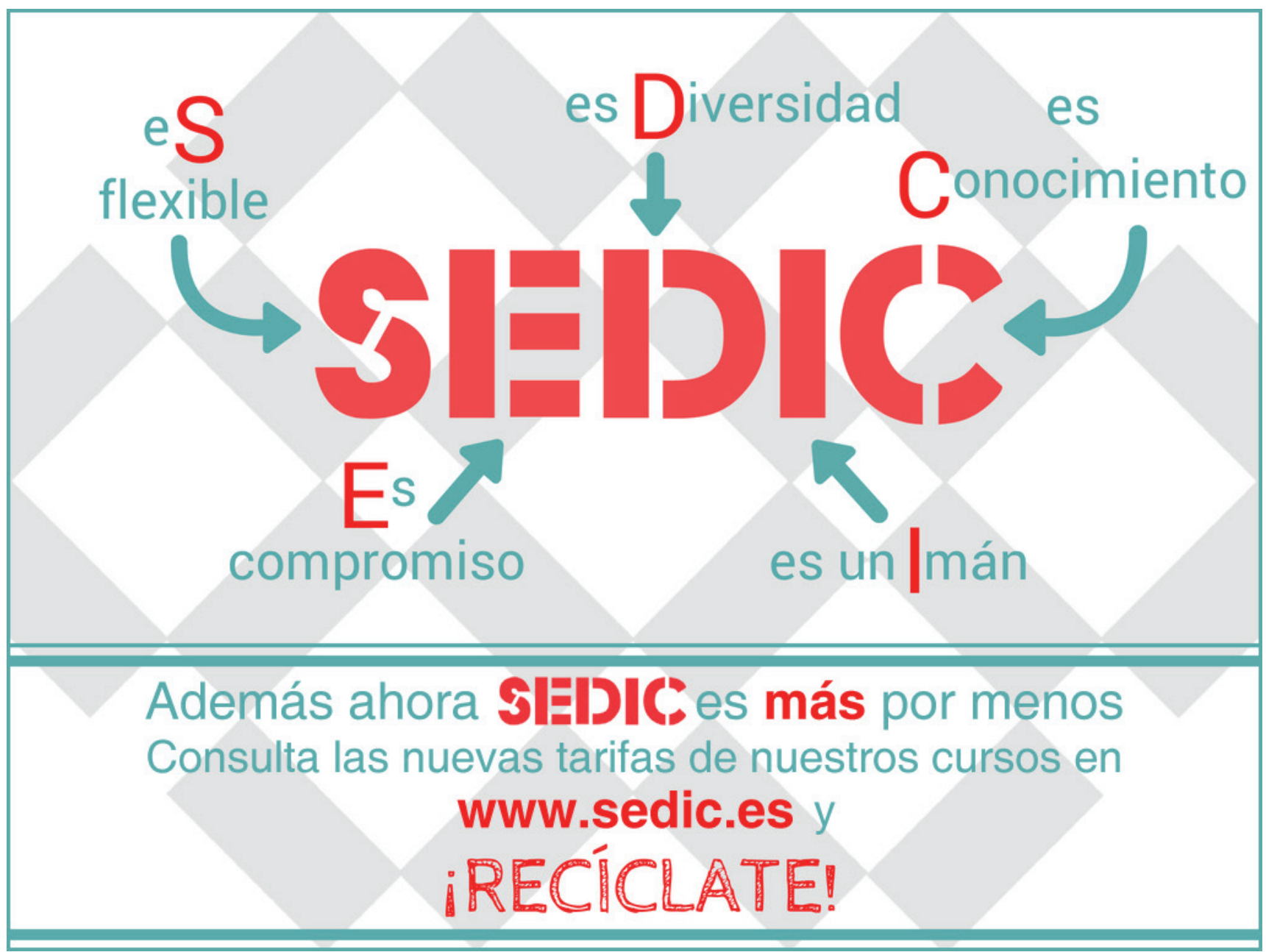

